\title{
Looking for New Strategies to Combat an Old Foe
}

\author{
Joshua P. Metlay, MD, PhD*
}

Division of General Internal Medicine and the Center for Healthcare Improvement and Patient Safety, Perelman School of Medicine, University of Pennsylvania, Philadelphia, Pennsylvania.

In the early part of the 20th century, pneumonia was a leading causes of death, particularly among older adults, for whom Osler termed it the Captain of the Men of Death. ${ }^{1}$ Mortality rates from severe (bacteremic) pneumonia were typically $80 \%$ to $90 \%$, and the introduction of antibacterial therapy in the 1940s reduced that mortality to $10 \%$ to $20 \%$. However, as pointed out by Austrian and Gold in a landmark paper in the 1950s, mortality for patients dying within the first 4 to 5 days was not reduced in the postantibiotic era. ${ }^{2}$ The survival rates for patients with severe community-acquired pneumonia minimally improved over the ensuing 50 years, despite the introduction of numerous new antimicrobial drugs and other medical interventions.

One promising area for therapeutic intervention relates to the potential adverse effects of the host inflammatory response in the setting of pneumonia. A growing body of literature supports the conclusion that the window of optimal host response may be relatively narrow. Too little response and patients quickly succumb to overwhelming sepsis. Too much response and a patient's hyperactivated inflammatory system can set off a cascade of secondary events, triggering events such as acute lung injury or ischemic heart disease. ${ }^{3}$ Studies have also established that the level of inflammation, as measured by biomarkers such as C-reactive protein, tumor necrosis factor, and interleukins, can identify patients at increased risk of adverse outcomes. ${ }^{4}$ Thus, it is logical to ask whether immune modulating therapies can improve outcomes for these patients.

In this issue of the Journal of Hospital Medicine, Shafiq and colleagues completed a systematic review and meta-analysis of corticosteroid therapy for patients with pneumonia. ${ }^{5}$ Updating prior reviews, they included 8 randomized controlled trials, all of which consisted of low-dose, systemic, steroid therapy as the intervention and standard care as the control arm. The overall quality of the included studies was

\footnotetext{
*Address for correspondence and reprint requests: Joshua P. Metlay MD, PhD, Perelman School of Medicine, University of Pennsylvania, 1232 Blockley Hall, 423 Guardian Drive, Philadelphia, PA 19104; Telephone: 215-898-1484; Fax: 215-573-0198; E-mail: jmetlay@upenn.edu

Additional Supporting Information may be found in the online version of this article.

Received: November 11, 2012; Accepted: November 20, 2012 2012 Society of Hospital Medicine DOI 10.1002/jhm.2005 Published online in Wiley Online Library (Wileyonlinelibrary.com).
}

judged moderate, and the overall size of the pooled data was only 1119 patients. In their analysis, adjunctive steroid therapy did not reduce in-hospital mortality, with 4 studies demonstrating effect sizes suggesting benefit, 3 studies demonstrating no benefit or harm, and 1 study favoring the nonsteroid arm. In these situations with grossly heterogeneous study results, it seems prudent to avoid overly interpreting pooled results, even if statistical tests for heterogeneity are nonsignificant. The investigators also reported a range of secondary outcomes, noting that hospital length of stay was significantly reduced in the pooled steroid treated arms.

The overall negative finding is clearly disappointing at a time when clinicians are looking for new treatments to improve outcomes for these patients. Pneumonia is a heterogeneous disorder, representing a wide range of microbial pathogens and underlying host risk factors. Current treatment guidelines for patients with community-acquired pneumonia are largely empirical and do not focus on pathogen identification, host risk factor analysis, or biomarker distributions to select antimicrobial therapy. ${ }^{6}$ In this regard, despite being 1 of the oldest conditions for which we have published guidelines for treatment, the treatment approach for pneumonia remains quite antiquated, ignoring recent advances in the incorporation of personalized treatment strategies for other illnesses. We may have reached the limits of one-size-fits-all treatment strategies for hospitalized adults with community-acquired pneumonia. To improve outcomes further, we need to understand the heterogeneity of the disorder and tailor therapies at an individual level. Rapid point-of-care tests for pathogens and host response offer the most promising approach toward this strategy.

It is notable that the majority of studies focus on in-hospital mortality, even though the impact of steroid therapy may be observed over a longer period of follow-up. Moreover, although mortality is clearly a relevant outcome, it is not the only patient-centered outcome of importance. However, other outcomes that are typically assessed, such as length of hospitalization and cost, are not patient-centered outcomes. These are process measures that reflect physician judgment as much as any patient response to treatment. We need to move the field forward by embracing patient outcomes beyond mortality to optimally evaluate new treatment strategies, particularly because the majority of patients will survive hospitalization for 
the illness. These outcomes would include time to resolution of major symptoms, such as cough and fatigue, and functional outcomes, including return to work and usual activities. Future comparative efficacy and effectiveness studies in pneumonia need to consider a much wider range of true patient outcomes. ${ }^{7}$

It is increasingly fashionable to adopt cross-disease approaches toward optimizing patient care, particularly in the hospital. Important initiatives that aim to reduce hospital injuries and improve transitions of care are relatively agnostic to specific disease states. Much of the research agenda of hospital medicine avoids a disease-specific focus, assuming such diseasespecific approaches are the domain of specialists. Yet, it is worth remembering that much of the progress for medical care can be traced to traditional considerations of disease pathophysiology and empirical studies of risk factors and treatments for specific disease. Hospitalists remain at the front line in dealing with most of the common illnesses that afflict patients. Battling those conditions 1 at a time should be an important component of the broader hospitalist research agenda. One hundred years after Osler charged the medical community to identify new strategies for treating an old enemy, we are still struggling to win the battle.

Disclosure: This work was supported in part by K24-AI073957 (JPM) from the National Institute of Allergy and Infectious Diseases, National Institutes of Health. The author has no conflicts of interest to report.

\section{References}

1. Osler W. The Principles and Practices of Medicine. 7th ed. New York, London: D. Appleton and Co.; 1909.

2. Austrian R, Gold J. Pneumococcal bacteremia with special reference to bacteremic pneumococcal pneumonia. Ann Intern Med. 1964;60:759-776.

3. Corrales-Medina VF, Serpa J, Rueda AM, et al. Acute bacterial pneumonia is associated with the occurrence of acute coronary syndromes. Medicine (Baltimore). 2009;88(3):154-159.

4. Kellum JA, Kong L, Fink MP, et al. Understanding the inflammatory cytokine response in pneumonia and sepsis: results of the Genetic and Inflammatory Markers of Sepsis (GenIMS) Study. Arch Intern Med. 2007;167(15):1655-1663.

5. Shafiq M, Mansoor M, Khan A, Sohail M, Murad M. Adjuvant steroid therapy in community-acquired pneumonia: a systematic review and meta-analysis. J Hosp Med. 2013.

6. Mandell LA, Wunderink RG, Anzueto A, et al. Infectious Diseases Society of America/American Thoracic Society consensus guidelines on the management of community-acquired pneumonia in adults. Clin Infect Dis. 2007;44(suppl 2):S27-S72.

7. Powers JH. Reassessing the design, conduct, and analysis of clinical trials of therapy for community-acquired pneumonia. Clin Infect Dis. 2008;46(8):1152-1156. 